\title{
¿CUALES SON LAS FORTALEZAS Y LOS OBSTACULOS DE LA RESPONSABILIDAD MEDICA EN EL ESTADO COLOMBIANO? ${ }^{1}$
}

\section{Jenny Paola García Osorio ${ }^{2}$}

\section{Resumen}

El presente artículo quiere dar a conocer algunos aspectos positivos y negativos que detenta la responsabilidad médica en el estado colombiano. Lo anterior, a causa de las continuas fallas que genera el servicio a la salud desde la constitución de 1991 y que se han reflejado en la comercialización del derecho a la salud, el manejo inadecuado de la tecnología, y en especial el declive de la relación médico - paciente, suministrando en el presente texto situaciones que deben ser reestructuradas para un funcionamiento correcto del sistema de la salud.

Ahora bien, se logró establecer en el texto que la responsabilidad médica en Colombia tiene un papel esencial en la vida de los ciudadanos, determinado no solo por la naturaleza jurídica de la responsabilidad médica, sino también, por elementos como la acción de repetición que fortalecen la responsabilidad del estado, haciendo énfasis y estableciendo la salud como derecho fundamental y como servicio público, además de ello, exponiendo las causas de la responsabilidad médica que no se encuentran exclusivamente en la acciones u omisiones del profesional de la salud.

El texto desarrollado permitió adelantar una investigación con enfoque cualitativo a través del método comparativo, por medio del cual se buscó conocer la fuente de algunas consecuencias que se presentan en la responsabilidad médica, distintas a la responsabilidad del profesional de la salud, así como la existencia de aspectos que promueven la responsabilidad médica en Colombia.

\footnotetext{
Abstract

${ }^{1}$ El presente artículo fue desarrollado con el propósito de optar por el título de especialista en derecho administrativo de la Universidad Santo Tomas.

${ }^{2}$ Abogada de la Universidad Los Libertadores, en curso especialización en derecho administrativo de la Universidad Santo Tomas. E-mail: jen07.07@hotmail.com.
} 
The present article wants to announce some positive and negative aspects that the medical responsibility holds in the Colombian condition. The previous thing, because of the continuous faults that the service generates to the health from the constitution of 1991 and that have been reflected in the commercialization of the right to the health, the inadequate managing of the technology, and especially the medical decline of the relation - patiently, supplying in the present text situations that they must be restructured for a correct functioning of the system of the health.

Now then, it achieved to establish in the text that the medical responsibility in Colombia has an essential paper in the life of the citizens, determined not only for the juridical nature of the medical responsibility, but also, for elements as the action of repetition that they strengthen the responsibility, doing emphasis and establishing the health as fundamental right and as public service, besides it, exposing the reasons of the medical responsibility that are not exclusively in her actions or omissions of the professional of the health.

The text previously developed allowed to advance an investigation with qualitative approach across the comparative method, by means of which one sought to know the source of some consequences that they present in the medical responsibility, different from the responsibility of the professional of the health, as well as the existence of aspects that promote the medical responsibility in Colombia.

Palabras Claves: Responsabilidad Medica, Derecho a la Salud, derecho a la vida, Médico, Paciente, falla del servicio.

Key words: Medical Responsibility, Law to the Health, Right to the life, Doctor, Patient, fault of the service.

\section{Introducción}

Actualmente en Colombia el ejercicio de la medicina se ha visto empañado por varias situaciones como son los constantes cambios en la legislación en materia de salud, contribuyendo de esta manera a la corrupción de un sistema que debería tener como única 
meta velar por que todo ciudadano que acceda al sistema de salud de una u otra forma vea protegido sus derechos a la salud y a la vida digna, siendo esto un resultado de una apropiada y eficiente atención por parte del profesional de la salud, del enfermero, del paramédico y de todo aquel servidor público que se encuentre en los llamados hospitales con denominación E.S.E, Empresas Sociales del Estado.

En contraste con la anterior descripción, encontramos que los problemas existentes que sufre el estado colombiano en el área de salud no solo tienen contribución de la corrupción, sino también problemas en los cambios de la ciencia, de la tecnología, el problema que abarca la globalización y la legislación entre otros, y estos son factores que conllevan a que la medicina se transforme, a que los profesionales de la salud no busquen eficazmente la mejoría del paciente o a que la salud sea negocio y que el paciente sea un objeto. Situaciones que se exponen en el presente artículo al observar que no todos los cambios que se han realizado han sido útiles y efectivos para la responsabilidad medica en Colombia

Siendo así, esta investigación busca exponer la importancia del manejo que se le ha dado a la actividad médica en Colombia basándonos en el estudio de sus fortalezas y debilidades, revelando las consecuencias que puede llegar a tener la población colombiana por la falta de conocimiento, consecuencias como la vulneración de sus derechos o el juzgar de manera desacertada las situaciones entre médico-paciente.

Ahora bien, comprendiendo las consecuencias que el desconocimiento del manejo de la actividad médica puede ocasionar, también es significativo exponer lo que puede generar un conocimiento por parte de la población colombiana sobre el manejo de la actividad médica, conocimiento que permitirá defender los derechos de la población, saber que existe el síndrome de burnout y entender de qué forma afecta la vida profesional, laboral y personal del médico como una de las causas esenciales de la responsabilidad médica en Colombia.

El estudio realizado permitió avanzar sobre la investigación de las causas, consecuencias y manejo de la responsabilidad médica en Colombia, captando en este texto las diferentes situaciones que se presentan en la responsabilidad medica en Colombia, situaciones que provocan reestructuración en hospitales, en reglamentos internos o un cambio en las creencias de los profesionales de la salud y de los pacientes. De ahí, que esta exploración de 
la responsabilidad medica en Colombia, confiere al lector bases para comprender no solo la problemática de la salud, sino también de los elementos positivos y negativos que tiene hoy la responsabilidad medica en Colombia.

\section{NATURALEZA JURÍDICA DE LA RESPONSABILIDAD MÉDICA}

"Según un principio tradicional del derecho, todo aquel que cause un daño a otro debe repararlo. Esa obligación de reparación se traduce en la responsabilidad del causante del daño, la cual puede ser según esa tradición de carácter penal o civil" (Rodríguez, 2013, p. 597).

El principio de la responsabilidad administrativa, que enuncia el párrafo anterior, señala que es obligación que el causante repare el daño, pero para ello se hace necesario que exista un nexo causal entre el hecho y el daño, dentro de la actividad medica ello quiere decir que, debe existir un nexo entre el acto médico y la producción del daño y además, debe tenerse presente y en cuenta las condiciones adversas, inmediatas o tardías producidas por las distintas situaciones que se llegan a presentar para que se pueda establecer una responsabilidad profesional médica.(Osorio, 2002 p.44 -45)

Ahora bien, dentro de la actividad médica no solo nos referimos a la acción realizada por el médico, sino también aquella realizada por enfermeras, auxiliares, clínica, hospitales, aseguradoras, EPS, IPS, etc., que a través de sus conductas o hechos realizados directa o indirectamente, causan determinados efectos jurídicos (Responsabilidad Médica, sf), uno de ellos lo es, la obligación de reparar y satisfacer las consecuencias de sus actos, omisiones y errores voluntarios o involuntarios dentro de ciertos límites cometidos en el ejercicio de su profesión. (Geosalud, sf).

De manera que "generalmente la responsabilidad medica no nace del incumplimiento de las obligaciones médicas, sino del cumplimiento defectuoso”. (Fernández, 2008 p. 65.) 
La importancia de la Responsabilidad Medica se halla contenida en los principios del estado social de derecho establecidos en la Constitución de 1991, empezando con lo plasmado en el artículo $2^{3}$ en el que se establece que dentro de los fines esenciales del estado "Las autoridades de la República están instituidas para proteger a todas las personas residentes en Colombia, en su vida, honra, bienes, creencias, y demás derechos y libertades, y para asegurar el cumplimiento de los deberes sociales del Estado y de los particulares", estos fines están estrechamente relacionados al servicio que brinda el médico desde el momento que toma el juramento de fidelidad profesional, ya que la actividad de la medicina busca siempre preservar la vida, honra, salud, dignidad, y libertades de todo ciudadano y se encuentra al servicio de la sociedad (Asociación Médica Mundial, sf).

Igualmente, la Constitución Política de Colombia en sus Artículos 48 y 49, manifiestan entre otras cosas que, "se garantizará a todos los habitantes el derecho irrenunciable a la seguridad social" (derecho a la salud y a saneamiento ambiental), que le "corresponde al estado organizar, dirigir, y reglamentar la prestación de servicio de salud a los habitantes. Así mismo, "Establecer las políticas para la prestación de servicios de salud por entidades privadas, y ejercer su vigilancia y control ${ }^{4}$,.

De acuerdo a los artículos antes expuestos, la Responsabilidad Médica encuentra un grado de importancia en temas de salud, y servicio público ya que, estos tienen influencia dentro del juramento hipocrático, el cual es una guía, dirección y parámetros para la actividad médica, así mismo ambos establecen preceptos, para la protección de los derechos fundamentales y prestación de servicio, respaldando en cierta medida la actividad médica y por ende estableciendo la responsabilidad médica.

\section{FORTALEZAS JURÍDICAS DE LA}

\footnotetext{
${ }^{3}$ Constitución Política de Colombia, 1991.
}

${ }^{4}$ Ibídem. 


\section{RESPONSABILIDAD MÉDICA EN COLOMBIA.}

Colombia por ser un estado social de derecho, tiene la obligación de velar y garantizar por los derechos de los ciudadanos establecidos en la Constitución Política de Colombia, y aún más por aquellos derechos considerados esenciales para la existencia digna de la persona, como lo es el derecho a la salud, el cual resulta ser "fundamental por ser universal, irrenunciable, inherente a la persona humana, integral e integrador, esencial para la materialización de una vida digna y con calidad" (Ministerio de Salud y Protección Social, sf), motivo por el cual debe ser el estado su máximo protector, garantizando el derecho fundamental a la salud con todas las garantías constitucionales y legales (Ministerio de Salud y Protección Social, sf).

Entre las garantías que brinda el estado, se encuentran aquellas que se implementa para proteger al ciudadano de las fallas ocasionadas por la prestación del servicio médico a cargo del mismo estado, motivo por el cual se desarrollaran algunas garantías constitucionales que son necesarias para guiar al médico en su diaria tarea de respetar, brindar trato igualitario, tener comunicación con el paciente, etc., sin olvidar que "relación médico-paciente es la piedra angular de la práctica médica y por lo tanto de la ética médica" (Asociación Medico Mundial, sf), quiere decir ello, que es esta relación médico paciente el punto de partida de la actividad médica y por ende de las fortalezas de la responsabilidad médica en nuestro país.

\subsection{La responsabilidad del estado desde el marco constitucional como fuente esencial para la responsabilidad medica en Colombia}

Considerando que la Constitución Política está llamada a garantizar la efectividad de los derechos fundamentales, la convivencia pacífica, y teniendo en cuenta el alcance de la gestión del Estado Colombiano, es necesario que se genere responsabilidad de quienes están encargados de ejercerla, así lo establece el artículo $90^{5}$ de nuestra constitución, al referirse que el estado deberá responder patrimonialmente cuando a causa de acciones u omisiones por parte de las autoridades públicas se vulnere el ordenamiento jurídico.

\footnotetext{
${ }^{5}$ Constitución Política de Colombia, 1991
} 
Así mismo, el Código de procedimiento Administrativo y de lo Contencioso Administrativo, establece que el Estado actuara y se desarrollara aplicando ciertos principios, entre ellos el mencionado en el numeral $7^{06}$ del Artículo $3^{\circ}$ que señala, “ las autoridades y sus agentes asumirán las consecuencias por las decisiones, omisiones o extralimitación de funciones, de acuerdo a la Constitución, las leyes y los reglamentos”.

Aunado a lo anterior, los distintos cambios que se dieron con la constituyente de 1991 permitieron que el Estado Colombiano comprendiera la importancia del derecho fundamental a la salud, haciendo posible que en la constitución 1991 se plasmara en el artículo $49^{7}$ que el estado "garantizara a todas las personas el acceso a los servicios de promoción, protección y recuperación de la salud”, además de ello se estableció que "corresponde al estado organizar, dirigir y reglamentar la prestación de servicio de salud a los habitantes ....".

El Estado implantó no solo la importancia del derecho a la salud, sino también la responsabilidad de la administración para garantizar el acceso adecuado a este derecho fundamental, que posteriormente se encargó de plasmar en la Ley 1751de 2015 en su artículo $5^{\circ}$ la obligación del estado de "respetar, proteger, y garantizar el goce efectivo del derecho fundamental a la salud”.

Con base en estos artículos, es que el estado colombiano cumple un papel importante dentro de las funciones del estado social de derecho, ya que su responsabilidad dentro de la actividad médica tiene una exigencia mayor siendo su función principal la prestación del servicio a la salud cuando se acredita que "la atención no cumplió con estándares de calidad fijados por el estado de arte de la ciencia médica", o se prueba "que el servicio médico no ha sido cubierto en forma diligente, esto es, que no se prestó el servicio con el empleo de todos y cada uno de los medios humanos, científicos, farmacéuticos y técnicos que se tengan al alcance" (Red Jurista, s.f.), son precisamente estas situaciones las que cuestionan la calidad y la prestación del servicio, observándose una falla por parte de un

\footnotetext{
${ }^{6}$ Código de procedimiento Administrativo y de lo Contencioso Administrativo

${ }^{7}$ Constitución Política de Colombia, 1991
} 
agente llámese médico o de una E.S.E. ${ }^{8}$, generando como resultado una responsabilidad médica.

\subsection{La protección del derecho fundamental a la salud y del servicio público a la salud como objetivo principal de la responsabilidad médica en Colombia}

Uno de los pilares del estado social de derecho en Colombia son los derechos fundamentales, los cuales tienen un papel importante frente a todo el aparato organizativo del Estado, sin estos perdería su objetivo, ya que los fines del estado se encuentran encaminados a promover y proteger los derechos establecidos en la carta política de Colombia (Corte Constitucional, 1992, p. 3).

Aunado a lo anterior, podemos decir que "los derechos fundamentales son verdaderos límites al poder" (Gil, 2013, p. 507), es por ello que el estado con el fin de proteger y garantizar de forma plena el derecho a la vida y dignidad humana, expidió la Ley 1751 de $2015^{9}$, mediante la cual se consagro la salud como un derecho fundamental al plasmar en su artículo $1^{\mathrm{o}}$ " la presente ley tiene por objeto garantizar el derecho fundamental a la salud, regularlo y establecer sus mecanismos de protección”, paralelamente estipulo en su artículo $2^{\circ}$ la naturaleza y contenido del derecho fundamental a la salud, mencionando que “de conformidad con el artículo 49 de la Constitución Política, su prestación como servicio público esencial obligatorio, se ejecuta bajo la indelegable dirección, supervisión, organización, regulación, coordinación y control del Estado”.

Lo anterior quiere decir que el derecho a la salud no solo es un derecho fundamental, sino que también se cataloga como un servicio público que tiene especial protección del estado y resulta indispensable para la actividad médica, al establecerse que "la protección Jurídica de la Salud y el respeto a la Dignidad Humana son las coordenadas básicas que regulan las cuestiones comprendidas dentro de la Responsabilidad Médica" (Geosalud, sf).

Es importante mencionar dos faceras del derecho a salud que se complementan, la primera de ellas la señala el artículo $49^{10}$ al mencionar que la prestación del servicio público a la

\footnotetext{
${ }^{8}$ Empresa Social del Estado.

${ }^{9}$ Ley Estatutaria No. 1751 del 13 de febrero de 2015 por medio de la cual se regula el derecho fundamental a la salud y se dictan otras disposiciones.

${ }^{10}$ Constitución Política de Colombia, 1991.
} 
salud que se realice a partir de la función administrativa, es un asunto de la responsabilidad patrimonial del estado y por consiguiente de la responsabilidad médica (Gil, 2013, p. 563), la segunda faceta se encuentra estipulada en la Ley 1751 de 2015 al regular la salud como derecho fundamental protegido por el estado, con el fin de que sea un "derecho inclusivo que se extiende no sólo a una atención médica oportuna y apropiada, sino que también a los determinantes implícitos de la salud, como el acceso a agua potable y segura, y saneamiento adecuado, condiciones ambientales y laborales saludables y acceso a la educación e información relativa a la salud, incluida la salud sexual y reproductiva"(Asociación Médica Mundial, sf).

Por consiguiente, se establece que al estado le corresponde una carga especial frente a la protección del derecho a la salud, "toda vez que las personas que se sometan a la praxis médica, quirúrgica y/o hospitalaria, lo hacen con la finalidad de que un grupo de personas con un conocimiento profesional y técnico brinden soluciones efectivas a situaciones que se relacionen de manera directa o indirecta con el concepto de salud" (Acosta, 2015, p 17), y como resultado de esa responsabilidad especial, el estado colombiano ha dispuesto elementos esenciales que se encuentran incluidos en las normas sobre derecho a la salud como por ejemplo, la accesibilidad, calidad, aceptabilidad, disponibilidad e idoneidad profesional, que conjuntamente son una garantía efectiva del derecho a la salud y una adecuada prestación del servicio público a la salud (Corte Constitucional, 2015).

\subsection{La acción de repetición un mecanismo de reparación patrimonial del estado en la responsabilidad médica colombiana}

La responsabilidad del estado frente a la actividad médica es tan amplia y compleja, que no solo se concibió al Estado como único responsable patrimonialmente, por el contrario, se menciona en varios artículos de nuestra constitución que la responsabilidad es la consecuencia de una omisión u acción por parte de agentes del estado, y de acuerdo a estos señalamientos es que hallamos que el legislador encuentra en la Acción de Repetición un "mecanismo judicial dispuesto por la Constitución Política, y desarrollado por la Ley,

para que el Estado recupere de sus servidores o ex servidores públicos o de los particulares que cumplen funciones públicas, los dineros que ha pagado por condenas 
impuestas en una sentencia, acta de conciliación u otro mecanismo alternativo de solución de conflictos a fin resarcir los daños antijurídicos causados. (Gil, 2013, p 708).

Lo anterior, teniendo en cuenta que el artículo $90^{11}$ de nuestra Constitución establece, que en aquellos casos en los cuales el Estado sea condenado patrimonialmente a la reparación, y esta condena sea consecuencia de conductas dolosas o gravemente culposas de un agente, el Estado deberá repetir contra este agente.

Así mismo, la acción de repetición ha sido desarrollada mediante la Ley 678 de $2001^{12}$, la cual reglamentó el ejercicio de la acción de repetición, manifestando en su artículo $2^{\circ}$ que esta acción es civil, de carácter patrimonial y sumado a lo ya expuesto establece que un "servidor o ex servidor público o el particular investido de funciones públicas podrá ser llamado en garantía dentro del proceso de responsabilidad contra la entidad pública, con los mismos fines de la acción de repetición, esto quiere decir que en aquellos casos en que un agente que presté el servicio público de la salud llámese enfermera, médico, etc., sea investigado como posible responsable por mala praxis ${ }^{13}$, al no haber sido diligente, no haber cumplido con los parámetros mínimos y estándares para enfrentar el caso clínico, y con ocasión a ello, el ciudadano que asiste a un hospital o centro hospitalario sufre una lesión o un detrimento mayor a su salud, el servidor público podrá ser llamado en garantía o a través de la acción de repetición de acuerdo a lo determinado en la Ley y la Constitución.

Las características de la acción de repetición la hacen única, es por ello que en el expediente 28.448, se define como " una acción de responsabilidad patrimonial que permite recuperar u obtener ante la jurisdicción el reembolso o reintegro de lo pagado por las entidades públicas en virtud del reconocimiento indemnizatorio impuesto judicialmente al Estado en una condena..." (Consejo de Estado, 2006), hay que mencionar además, que

\footnotetext{
${ }^{11}$ Constitución Política de Colombia, 1991.

12 Por medio de la cual se reglamenta la determinación de responsabilidad patrimonial de los agentes del Estado a través del ejercicio de la acción de repetición o de llamamiento en garantía con fines de repetición.

13 Término que se utiliza para referirse a la responsabilidad profesional por los actos realizados con negligencia. La forma más conocida de la mala praxis es la negligencia médica o mala praxis. En medicina una mala praxis se utiliza para indicar una mala gestión "por acción o por omisión”.
} 
otra de las características es que goza de obligatoriedad, y el incumplimiento a este deber se constituye una falta disciplinaria ${ }^{14}$.

Su importancia, por ende, no radica únicamente en el reembolso o reintegro de una suma de dinero, sino también en que es "un mecanismo procesal autónomo, no accesorio a otra acción o proceso, solo que condicionó su precedencia al cumplimiento de ciertos requisitos” (Gil, 2013, p 712).

En este punto es apropiado señalar que los requisitos a los que se hizo alusión, son los siguientes: existencia de una condena en contra de la entidad estatal, pago realizado por parte de la Administración, calificación de la conducta del agente como dolosa o gravemente culposa, calidad del agente del estado y de la conducta desplegada por este (Procuraduría General de la Nación, sf). De los elementos citados no existe una escala de importancia, pero si se hace preciso indicar que la acción u omisión del servidor público cuenta con un "análisis subjetivo de la conducta, para determinar si actuó con dolo o culpa grave, pues de no ser así, aunque se haya declarado la responsabilidad del Estado por los hechos, no procede condena en contra de su agente” (Gil, 2013, p 703), ya que es requisito indispensable que el estado haya sido condenado por la conducta dolosa o gravemente culposa de funcionarios y empleados (Gil, 2013, p 730).

La jurisprudencia colombiana, es consciente que la actividad médica es de riesgo ya que a diario se presentan innumerables acciones entre las que encontramos: valoraciones, diagnostico, toma de exámenes, terapias, intervenciones quirúrgicas etc., pero "en las que un grupo de personas con un conocimiento profesional y técnico buscan brindar soluciones efectivas a estas situaciones" (Consejo de Estado, 2007), sin lograr en muchas situaciones un resultado satisfactorio para el paciente, por el contrario la administración evidencia una falla, un funcionamiento anormal, negligente o un descuido del servicio médico, provocando con ello un daño irreversible de lo cual resulta que el estado puede ser condenado responsable patrimonialmente y por consiguiente, repetir contra el agente que prestó el servicio a la salud (Consejo de Estado, 2007).

\footnotetext{
${ }^{14}$ Ley 678 de 2001, artículo 4ㅇ․
} 
Un ejemplo de la acción de repetición de acuerdo a lo planteado en el párrafo anterior, es el daño irreversible que puede ser causado por un error grave del personal médico, como es una auxiliar de enfermería, que con ocasión del servicio que presta se ofrece voluntariamente a efectuar curación a un menor, vertiéndole formol sobre la cabeza, produciéndole graves quemaduras en ambas córneas, de lo que se derivó su ceguera irreversible, por lo que el Consejo de estado ante esta situación no solo llamo en garantía a una de las entidades, sino que mediante sentencia declaro responsable administrativamente a la nación, y la condeno solidariamente junto con el Hospital, el Ministerio de Salud y la auxiliar de enfermera, buscando de esta manera la sección tercera precisar la responsabilidad solidaria, claro está, que en dicha sentencia se hizo salvedad indicando que "la entidad pública mencionada, deberá cancelar las sumas de la correspondiente liquidación de perjuicios, para luego repetir por los respectivos valores en contra de su funcionaria o empleada" (Consejo de Estado, 2007).

Cabe aclarar, que el juez previo a declarar responsable al estado o declarar la responsabilidad solidaria (Responsabilidad del Centro Hospitalario y del agente), debe realizar un estudio concienzudo en el que se "debe tener en cuenta las características particulares del caso que deben armonizarse con lo previsto en los artículos $6^{\circ}$ y 91 de la Constitución Política sobre la responsabilidad de los servidores públicos, (...) haya establecido expresamente que el deber de las entidades estatales de repetir contra sus funcionarios o ex funcionarios, sólo surge en la medida en que el daño a cuya reparación patrimonial hayan sido condenadas" (Procuraduría General de la Nación, s.f).

Con la acción de repetición, no se le está sancionando al médico, auxiliar de enfermería, o cualquier otro servidor público que practique activamente la medicina, toda vez que lo que se busca es la reparación patrimonial del Estado, esta acción es un mecanismo indemnizatorio, de carácter público "y su finalidad es la protección del patrimonio y de la moralidad pública, y la promoción del ejercicio de la función pública con eficiencia" (Gil, 2013, p 713).

\subsection{Ley ética médica como mecanismos fundamentales que garantiza $y$ fortalece la relación médico - paciente.}


Actualmente la ética médica se encuentra estipulada en la Ley 23 de 1981 la cual establece normas que promueven el cumplimiento y protección de los derechos fundamentales de los pacientes, por consiguiente, hallamos que en su artículo $2^{\text {o15 }}$, se fomenta la igualdad, el respeto por la dignidad humana y la vida, así mismo busca sensibilizar el aspecto humano de la enfermedad. (Ministerio de Salud, sf).

Lo anterior, es relevante ya que la protección de los derechos del paciente, resultan indispensables para un equilibrio entre la relación Médico - Paciente, es así como la Ley de ética médica hace alusión al consentimiento informado, al manejo que se le brinda a la historia clínica como mecanismos que garantizan un óptimo servicio y protección de los derechos fundamentales.

\subsubsection{El consentimiento informado garantía para el respeto de los derechos de los pacientes en la responsabilidad médica en Colombia.}

En los últimos años los cambios en el campo de la medicina se han hecho más visibles, más significativos, dejando de lado el paternalismo hipocrático y abriendo paso a una nueva brecha en la que el paciente "espera ser curado, que se le suministré la información requerida, que ésta sea suficiente y veraz y que le facilite decidirse de lo que de verdad sea bueno" (Ilustrados, sf), es por ello que el consentimiento informado se constituye para los pacientes como "un derecho que le permite conocer el alcance y las posibles consecuencias médicas de sus decisiones" (Sura, sf).

De otro lado, encontramos que el profesional de la salud tiene entre sus obligaciones, el deber de informar plenamente al paciente "sobre los riesgos y las posibles complicaciones

\footnotetext{
15

5 ARTICULO 2o. Para los efectos de la presente ley, adóptense los términos contenidos en el juramento aprobado por la Convención de Ginebra de la Asociación Médica Mundial, con la adición consagrada en el presente texto. El médico deberá conocer y jurar cumplir con lealtad y honor el siguiente Juramento médico: Prometo solemnemente consagrar mi vida al servicio de la humanidad; Otorgar a mis maestros el respeto, gratitud y consideración que merecen; Enseñar mis conocimientos médicos con estricta sujeción a la verdad científica y a los más puros dictados de la ética; Ejercer mi profesión dignamente y a conciencia; Velar solícitamente y ante todo por la salud de mi paciente; Guardar y respetar los secretos a mí confiados; Mantener incólumes, por todos los medios a mi alcance, el honor y las nobles tradiciones de la profesión médica; Considerar como hermanos a mis colegas; Hacer caso omiso de las diferencias de credos políticos y religiosos, de nacionalidad, razas, rangos sociales, evitando que éstas se interponga entre mis servicios profesionales y mi paciente; Velar con sumo interés y respeto por la vida humana, desde el momento de la concepción y, aun bajo amenaza, no emplear mis conocimientos médicos para contravenir las leyes humanas; Solemne y espontáneamente, bajo mi palabra de honor, prometo cumplir lo antes dicho.
} 
de un tratamiento o intervención” (Sura, sf), ello quiere decir, que la aplicación de la ética profesional para el médico constituye "una nueva actitud frente al paciente, derivada del concepto de responsabilidad profesional, que implica una serie de riesgos que deben evitarse mediante una continua mejora en sus conocimientos técnicos y una nueva consideración de la relación médico paciente que ponga énfasis en el consentimiento informado" (Ilustrados, sf).

El médico no debe olvidar que la creación, aplicación y desarrollo de nuevas tecnologías en las ciencias de la salud, han concebido una nueva actitud frente al paciente, al señalar que "el paciente es el verdadero protagonista de la relación médico-paciente", y aunque parte de la sociedad, instituciones y el mismo paciente no le encuentren relevancia al consentimiento informado, la obtención de éste es vital, ya que es un "proceso que va más allá de obtener una firma sobre un formulario estandarizado" (Scielo, sf), es comprender que el paciente es titular de derechos, que es capaz y por consiguiente puede aceptar o rechazar un determinado tratamiento (Scielo, sf).

En Colombia, el consentimiento informado en la relación médico-paciente representa una nueva relación "en la que se exige un mayor respeto a las personas del que se tenía hasta ahora" (Ilustrados, s.f), en la que los conocimientos no solo éticos sino jurídicos del médico, recogen mayor relevancia en su práctica profesional en el momento de contacto con su paciente, de allí que se origine un "auténtico momento de la verdad en la relación médico-enfermo y cuya trasgresión puede originar situaciones potenciales de responsabilidad, derivadas no tanto de la mala práctica profesional, sino del desconocimiento de aquellos aspectos que son sustanciales en la relación médico-paciente desde la perspectiva de la Ética y del Derecho" (Ilustrados, s.f).

Respecto a lo mencionado previamente, el consentimiento informado no solo es parte esencial de la ética médica al consagrar en el artículo 15 de la Ley 23 de 1981, que el profesional de la salud pedirá consentimiento al paciente en aquellos tratamientos médicos y quirúrgicos que se le considere indispensable, sino que también responde a una obligación legal en la que se le exige informar de "manera clara, oportuna y completa sobre los riesgos de las intervenciones" (Scielo, s.f), a su vez, la norma ha previsto que en eventos como, i) el estado mental del paciente no es normal, ii) el paciente se encuentra en 
estado de inconciencia, iii) el paciente es menor de edad, la obligación de informar se aplique a los responsables del paciente (familiar, allegado o representante) a quienes se les explicara de manera anticipada las consecuencias de la intervención (Scielo, s.f).

Parte de la premisa del consentimiento informado es el cambio del esquema tradicional paternalista al fundado por el respeto a la autonomía del paciente, el cual facilita y protege la relación médico - paciente, fomenta la actividad medica hacia una práctica y comunicación más humanizada (Scielo, s.f), igualmente fortalece las destrezas y competencias para así evitar errores en la atención de la salud y disminuir la posibilidad de eventos adversos (Ministerio de Salud, s.f).

De modo que, para la construcción, sostenibilidad y protección de la relación médico paciente que existe en Colombia es elemental el consentimiento informado, dado que se considera "un acto clínico cuyo incumplimiento puede generar responsabilidad y es la mejor expresión de una adecuada relación médico-paciente entendida como un contrato reciproco de derechos y deberes, responsable, leal y auténtico" (Ilustrados, s.f).

La esencia, así como la importancia del consentimiento informado se encuentra fundado en el respeto por la autonomía del paciente, constituyéndose como el mejor instrumento para facilitar y proteger la relación médico - paciente, que, asociado a los valores y principios morales de la ética normativa, promueven y protegen los derechos fundamentales. (Ilustrados, s.f).

\subsection{2. la historia clínica un acto médico obligatorio que garantiza una atención médica de calidad y promueve la relación médico - paciente en la responsabilidad médica en Colombia}

En el ejercicio de la medicina la relación médica - paciente no se limita al consentimiento informado, también se requiere de otros procedimientos médicos que les permitan a los profesionales de la salud brindar con mayor garantía y calidad el servicio prestado, por lo que encontramos que la historia clínica juega un papel notable al ser reconocida como "un documento privado, de tipo técnico legal obligatorio y sometido a reserva, en el que se registran cronológicamente las condiciones de salud del paciente, los actos médicos y los 
demás procedimientos ejecutados por el equipo de salud que interviene en su atención” (Hospital Universitario San Ignacio, sf).

Por consiguiente, la historia clínica no solo es relevante para los profesionales de la salud o el paciente, este procedimiento por ser de tipo legal obligatorio entra a ser parte de los actos médicos que se encuentran respaldados y protegidos por la ley, "convirtiéndose en la prueba documental que evalúa el nivel de la calidad asistencial en circunstancias de reclamaciones de responsabilidad a los profesionales sanitarios y/o a las instituciones públicas" (Geosalud, s.f).

Su obligatoriedad radica principalmente en la información que guarda con relación a la atención prestada al paciente, la cual permite conocer la existencia o no de una mala praxis, al establecer en su contenido cómo ha evolucionado el padecimiento por el cual el paciente acudió al médico, antecedentes familiares y personales, patológicos y quirúrgicos, exámenes físicos, igualmente sustenta un diagnóstico y un tratamiento (Encolombia, s.f).

Para que el contenido de la historia clínica sea de utilidad, es necesario un correcto diligenciamiento el cual debe hacerse en forma clara, legible, sin tachones, sin utilizar siglas, que las anotaciones realizadas conserven fecha, hora y nombre completo junto con la firma del profesional de la salud, técnicos y auxiliares que realizan la atención clínico asistencial directa al paciente (Encolombia, s.f), lo que permite garantizar "no sólo la práctica médica o acto médico, sino también el cumplimiento de algunos de los principales deberes del personal sanitario respecto al paciente: deber de asistencia, deber de informar, etc.," (Grupo EMI, s.f).

De ahí que, la omisión de información o registro de procedimientos que nunca se efectuaron en la historia clínica, tenga graves consecuencias empezando con la responsabilidad médica por falla en el servicio, seguido del delito de falsedad ideológica en documento privado, ya que "Todo lo que no se precise en ella puede ser usado en contra de quien cometió la omisión, pero adicionalmente se expone a las sanciones ante el Tribunal de Ética Médica, disciplinariamente" (Encolombia. sf), y si dicha omisión es ejecutada por un médico, enfermera, auxiliar y este es un empleado oficial se está ante el delito de prevaricato por omisión (Encolombia. s.f). 
Como hemos plasmado, la historia clínica no son simples documentos, exámenes e información que se requieren sin fin alguno, por el contrario "es un documento de vital importancia para la prestación de los servicios de atención en salud y para el desarrollo científico y cultural del sector" así lo plasmo el estado colombiano en la resolución 1995 de 1999 del Ministerio de Salud y Protección Social, por la cual se establecen las normas para el manejo de la historia clínica.

De modo que la importancia de ese documento vital llamado historia clínica, reside en que permite "entablar una relación más personal con el paciente" que puede "ir ayudando poco a poco a ver más claro cuál es el problema del paciente y con eso ir planteando un tratamiento correcto" (Mundo Gestalt, sf) y ello lograr una efectiva y correcta prestación del servicio de atención en salud.

\section{DEBILIDADES JURIDICAS DE LA RESPONSABILIDAD MÉDICA EN COLOMBIA}

Las continuas reformas al sistema de salud no dignifican la actividad médica, por el contrario, su labor social desaparece y en su lugar encontramos individuos robotizados frente al dolor y preocupación del paciente, lo que muestra un servicio público de la salud deshumanizado en el que el paciente se convierte en "número de cama o historia clínica" (Periódico La Nación, sf), y los profesionales de la salud a causa de las largas jornadas, de la frustración laboral, profesional y personal olvidan ser solidarios, respetuosos y comprensibles, convirtiéndose en individuos que "hablan con autoritarismo" y al paciente lo tratan "como si los estuvieran regañando o juzgando, en lugar de servir como apoyo ante la necesidad que estos tienen de sus servicios y la obligación de los mismos de atenderlos” (Periódico La Nación, sf).

Hay que mencionar además, que el problema de la responsabilidad médica no radica únicamente en la relación médico - paciente, o en la prestación indebida por omisión u acción del servicio público que presta el profesional de la salud, se genera también, por la administración del estado que se enfoca de forma errónea al no alcanzar una administración eficiente, "donde al tener un orden administrativo claro, puede llevar a la consecuencia de una atención adecuada a los usuarios” (Noticias Universia Colombia, sf). 


\subsection{La falla del servicio médico por parte de la admistraciòn como factor determinante en la responsabilidad médica en Colombia.}

La falla del servicio como elemento principal de la responsabilidad médica estatal en Colombia, "es una inadecuada prestación de un servicio prestado por la administración" (Universidad Nacional Abierta y a Distancia, sf), ello quiere decir que, la prestación del servicio médico "no cumplió con estándares de calidad fijados por el estado del arte de la ciencia médica, vigente en el momento de la ocurrencia del hecho dañoso" (Consejo de Estado, 2011, p.1).

Es evidente que el sistema de salud colombiano tiene muchos quiebres, y lo más grave es que "la atención en salud no parece mejorar, cometiéndose las mayores violaciones a los derechos de las personas, en tanto se tiene un sistema inoperante plagado de malas administraciones" que lo único que concibe es "la mala experiencia de acceder a la salud en Colombia" (Senado de la República - Sala de Prensa, sf), sentimiento que no es desconocido para los colombianos.

Por lo que aquella falla del servicio que ocasiona la responsabilidad médica del estado, no es otra que la falla de la administración frente a los recursos, políticas y modelos aplicados a la salud, que al delegar funciones obstruyen el acceso oportuno y de calidad a éste, en el que "los principales perjudicados siguen siendo los usuarios, de los que ni siquiera se salvan aquellos que pertenecen a modalidades de medicina pre pagada o planes complementarios" (Senado de la República - Sala de Prensa, sf).

El estado por su parte, sabe que es responsable de "garantizar un servicio de salud que respete unos determinados estándares de calidad" (Ministerio de Salud y Protección Social, sf), que deben garantizar la salud como derecho fundamental y como servicio público, pero la realidad es otra, ya que no le ofrece al ciudadano un servicio de calidad y con calidez, por el contrario, está interesado en el crecimiento económico del negocio de la salud.

Razón por la cual, el manejo inadecuado de la administración, es la causa fundamental de la responsabilidad médica, puesto que el estado ha olvidado que "las instituciones 
prestadoras de salud requieren de manera primaria la inversión en parte administrativa antes que asistencial para edificar un buen proyecto" (Noticias Universia Colombia, sf ) y de esta manera contar con personal idóneo en la administración de los E.S.E ${ }^{16}$ y en las diferentes áreas de la salud, para brindar un servicio público que vele por la salud, vida y dignidad de los pacientes en todo procedimiento.

Además, se considera que la falla del servicio por parte de la administración se concentra en la constante competencia de las Empresas Sociales del Estado y en la administración indebida de recursos que terminan por contribuir a la crisis de la salud, (Noticias Universia Colombia, sf) aunado a ello encontramos un sistema de salud corrupto, que lo último que le interesa es la responsabilidad y correcta aplicación de la ética profesional ('Periódico El Espectador, sf), olvidando que el fin de la medicina es "cuidar de la salud del hombre y propender por la prevención de las enfermedades, el perfeccionamiento de la especie humana y el mejoramiento de los patrones de vida de la colectividad, sin distingos de nacionalidad, ni de orden económico-social, racial, político y religioso". ${ }^{17}$

Es evidente que la administración ha eludido la misión de la medicina, para dar paso a metodologías, reglas, tecnologías y procedimientos que impiden que el medico cumpla con su labor profesional y social, siendo así, tanto el profesional de medicina, como los demás individuos que prestan el servicio público de la salud se van volviendo negligentes e inhumanos ante las diferentes necesidades del paciente, incrementando "los errores médicos con consecuencias muy negativas en la morbilidad, mortalidad y costos de atención” (Scielo, sf), terminando por perjudicar la salud, vida, integridad y dignidad del paciente.

De manera que se hace necesario cambiar el chip para evitar que esta crisis sea permanente, se debe empezar por apreciar de manera distinta la calidad de atención al paciente al considerarlo "no sólo como un problema médico gerencial, sino como un problema moral y, como tal, estar sustentado en una educación en valores que garantice la participación

\footnotetext{
${ }^{16}$ Establecimiento social del estado

${ }^{17}$ Artículo 1을 Ley 23 de 1981. Por la cual se dictan normas en materia de ética médica.
} 
activa de todos los actores del sistema, siendo la excelencia, la solidaridad y el profesionalismo valores fundamentales" (Scielo, sf).

Ahora bien, este cambio se debe gestionar aprendiendo de los errores, por lo que no se puede olvidar que a causa de la falla del servicio de la administración, el paciente puede perder " la oportunidad que tenía de mejorar o recuperar su salud" (Consejo de Estado, 2011, p. 1), como es el caso de la muerte del paciente por demora en intervención quirúrgica, la muerte de un bebe que estaba por nacer, quemaduras en corneas de un menor, entre otras situaciones que son consecuencia de las modalidades de medicina, de la delegación de funciones a personal no capacitado, disminución de personal, bajo presupuesto, corrupción estatal, etc.

Es inevitable advertir que de continuar la falla del servicio por parte de la administración, se estaría desatendiendo jurídicamente el derecho fundamental a la salud, el servicio público de la salud y el respeto a la dignidad humana como coordenadas esenciales que regulan la Responsabilidad Médica en Colombia. (Geosalud s.f.).

\subsection{Síndrome de BURNOUT, impedimento para una relación médico - paciente satisfactoria en la responsabilidad médica en Colombia.}

La relación médico - paciente cada día se fractura más, factores hay muchos, pero entre ellos se destaca uno que acoge un conjunto de elementos esenciales que se han perdido y que son necesarios para una relación con calidad y calidez, estamos refiriéndonos al síndrome de Burnout, un "tipo de estrés laboral e institucional generado en profesionales que mantienen una relación constante y directa con otras personas (médicos, enfermeros, profesores etc.) que se refleja en una "sensación de fracaso, existencia agotada o gastada que resulta de sobrecarga por exigencia de energías, recursos personales o fuerza espiritual del trabajador" (Universidad del Rosario, sf).

Esa sensación de fracaso o desgaste emocional y físico que pueden llegar a tener los médicos, los especialistas, enfermeros, se encuentra reflejado en el trato inhumano constante que reciben los pacientes en nuestro país, también es cierto que el pensamiento de la sociedad en general es la del médico inhumano e intolerante, pero pocas veces se piensa 
en que éste individuo al que se busca cuando existe un percance en nuestra salud, también siente frustración ante diferentes situaciones laborales y personales, como es el ver fallecer a un paciente o el sentirse frustrado por no ver progreso en su vida profesional, ese conjunto de situaciones que perjudican al médico terminan por afectar el trato que recibe el paciente, ya que recibe "indiferencia y actitudes distantes" ello quiere decir que," no hay una implicación personal positiva en el proceso de la tarea" (Psicoayuda, sf).

En Colombia, es poco conocido el síndrome de Burnout, desconociendo de esta manera que el principal detonante es el entorno laboral y las condiciones de trabajo que se le presentan al profesional de la salud, situaciones que se generan por la carga excesiva del trabajo, altos niveles de estrés, falta de formación para efectuar las funciones, malas relaciones y grandes exigencias en su entorno laboral (DMedicina, sf), escenarios que sí son evidentes para la comunidad colombiana de manera individual pero no como un conjunto de realidades que constituyen "un trastorno emocional" (DMedicina, sf).

Para evitar caer en el error antes mencionado, es necesario conocer las diferentes manifestaciones del síndrome de burnout, la primera de ellas es el agotamiento emocional, que se ve reflejado en la pérdida de energía, fatiga a nivel físico y psíquico y se ocasiona por funciones laborales permanentes, que le generan impotencia como es en el caso del médico al perder un paciente en un quirófano, la segunda manifestación es la falta de realización personal, aquella hace referencia a los sueños, metas que como persona el médico desea, y al no llevarse a cabo su autoestima disminuye, llega la frustración y el estrés fisiológico y cognitivo, por último la despersonalización, en esta manifestación se funda ese trato indebido que reciben los pacientes, ya que el profesional de la salud expresa su estrés en su comportamiento negativo hacia los usuarios, su nivel de irritabilidad aumenta igual que su pérdida de motivación, y como resultado de ello, sus relaciones cotidianas llegan a la deshumanización en el trato (Psicología y Mente, s.f).

Se podría creer que el síndrome de burnout solo debería afectar la órbita personal del médico, no traspasar lo profesional o laboral, pero se nos olvida como ciudadanos que el profesional de la salud por sus largas jornadas pasa la mayor parte del tiempo en el hospital, por lo que su estrés, depresión e irritabilidad llega a sus compañeros y pacientes, de ahí parte la importancia de conocer y prevenir éste síndrome, para evitar que el servicio público 
que presta el médico sea deficiente, y que la relación médico - paciente sea defectuosa, por tanto se debe empezar por reconocer que el síndrome de burnout "es un proceso secundario a una respuesta negativa al estrés laboral crónico"(Medigraphic, sf).

Simultáneamente, se debe hacer lo necesario para mejorar la organización de la entidad con el fin de proveerle al médico herramientas adecuadas para que su tarea sea desarrollada de forma apropiada, disminuir su sobrecarga laboral, optimizar espacios de comunicación con los compañeros y hacerle saber que su convicción es importante, aunado a ello, buscar un punto intermedio que le permita al profesional de la salud ver en su labor una oportunidad para aprender y crecer en otros ámbitos diferentes (DMedicina, sf).

Lo anterior, para ayudar de forma positiva y "significativamente en el desempeño laboral del personal de salud" (Medigraphic, s,f), sin olvidar que "la intervención tanto individual como grupal dirigida a la prevención del síndrome, así como la promoción del tratamiento integral en todas sus dimensiones" (Scielo, sf) es fundamental para permitir que los profesionales de la salud realicen "su trabajo en óptimas condiciones de calidad, eficiencia y satisfacción personal" (Scielo, sf) y por ende la relación médico-paciente sea satisfactoria.

\subsection{La deshumanización como consecuencia de la tecnología moderna aplicada a la actividad médica en Colombia.}

El servicio público que prestan los médicos al buscar salvaguardar la vida y la salud de los ciudadanos es una acción que requiere de profesionalismo, vocación y ética, factores que se han venido perdiendo a causa de la globalización, ya que "la práctica médica actual, estrechamente asistida por el avance técnico y científico, detenta como nunca antes un insospechado poder sobre las personas” ( Centro de Bioética Juan Pablo II, sf) olvidando que está "llamada a ser: el servicio más noble, honorable y dignificante" ( Centro de Bioética Juan Pablo II, sf).

Muchos médicos olvidan que la calidad del servicio que prestan se mide en la satisfacción del paciente, y este sentimiento de satisfacción empieza por reconocer que el paciente es "un ser humano investido de dignidad absoluta" y no como alguien que "podría representar una inclinación favorable o no, de alguna tasa o índice estadístico" (Centro de 
Bioética Juan Pablo II, sf), esta práctica hoy en día es más común de lo que se quiere reconocer en los hospitales del estado

En nuestro país, el objetivo social e hipocrático en la profesión de la medicina, se ha perdido por el mérito individual que puede llegar a lograr un médico a través de instrumentos tecnológicos y científicos (Federación Médica Colombiana, sf), perdiendo por ende el objetivo primordial de esta profesión, de ahí que "la sociedad actual manifiesta su inconformidad cada vez en forma más frecuente, con desafortunada agresividad, contra la medicina y especialmente contra los médicos" (Federación Médica Colombiana, sf), provocando que el paciente no crea que el médico tiene una ética profesional.

La profesión de la medicina está en una crisis humanística enorme, considerando que el médico "no puede ni debe desconocer que antes que médico es un ser humano, y que el paciente también lo es, entonces la relación entre médico y enfermo debe efectuarse siempre desde un plano humano" (Dr. Mario Fiore Columna Vitae, sf), y es precisamente esta última parte la que el profesional de la medicina ha perdido incluso desde que inicia sus conocimientos en una institución universitaria.

Esta crisis que causa deshumanización como consecuencia de la tecnología moderna, se refiere a la "pérdida relativa de un conjunto de atributos o virtudes deseables en el medico que presta la atención en salud en su práctica diaria" (Federación Médica Colombiana, sf), estos atributos son conocidos por los estudiantes de medicina, pero en el momento que se realiza el juramento hipocrático se convierte en guía para el médico, que debería, por consiguiente "auxiliar, a socorrer a pacientes, logrando entonces un sentido más pleno de la vida en general y de la propia vida” (Dr. Mario Fiore Columna Vitae, s.f).

Aunque la tecnología médica moderna es impresionante, permitiendo la detección oportuna de muchas enfermedades, intervenciones quirúrgicas optimas, prolongación de vida etc., también es cierto que la tecnología en la práctica moderna de la medicina se olvida de la calidad de vida de los pacientes, impide un acceso igualitario, sobrecarga de los sistemas de salud, evaluación constante en la actividad médica relación costo - beneficio (Instituto Técnico para la Acreditación de Establecimientos de Salud - ITAES ${ }^{18}$, s.f), razones por los

\footnotetext{
${ }^{18}$ Impacto de la Tecnología en la práctica de la medicina.
} 
cuales en muchas oportunidades se considera la medicina actual un negocio lucrativo y no un servicio público.

La aplicación de la tecnología moderna en la actividad medica se ha caracterizado por la “alienación y despersonalización, cambios sociales relacionados con una mayor burocratización y urbanización, nuevas modalidades de comunicación, pérdida de la privacidad" (Instituto Técnico para la Acreditación de Establecimientos de Salud ITAES $^{19}$, s.f), situaciones que conllevan a la deshumanización de la medicina o quiere decir ello a la ruptura en la relación médico - paciente.

No hay duda que los avances tecnológicos en materia de medicina han permitido mayor probabilidad de supervivencia, pero la confiabilidad del profesional de la salud en estos instrumentos es alta, ocasionando errores fatales que perjudican la vida, salud e integridad del paciente, quedando demostrado que en ocasiones el paciente es "un objeto de explotación por la todopoderosa industria de la salud y no un fin en sí mismo en consideración al respeto que se merece como persona” (Academia Nacional de Medicina en Colombia, s.f.).

Respecto a lo anteriormente mencionado, se tiene que en el campo de la medicina los actores han aumentado y no precisamente para favorecer la relación médico - paciente o para hacer de la medicina una actividad mucho más valiosa de la que era hace siglos, por el contrario, la llegada de empresarios, economistas, administradores e intermediarios denigro este servicio público convirtiéndolo en un negocio en el que "los médicos han visto restringida su responsabilidad, su autonomía, su ejercicio profesional y en fin se han convertido en funcionarios de instituciones públicas o privadas que son las que organizan y manejan la atención de salud y están sometidos, por supuesto a sus reglas y restricciones porque unas y otras tiene afán de lucro o de rentabilidad financiera", (Federación Médica Colombiana, s.f), no hay que olvidar que las instituciones públicas que prestan el servicio de la salud deben ser rentables, tener reglas, y tener procedimientos para atender al llamado usuario, que debe por supuesto estar registrado para ser atendido y debe esperar por orden de urgencia esta atención prioritaria, a esto se llega con la deshumanización de la medicina.

\footnotetext{
${ }^{19}$ Impacto de la Tecnología en la práctica de la medicina.
} 
Sumado a lo anterior, tenemos que el estado colombiano como administrador de la salud es el causante en gran medida de esta situación en la que los profesionales y estudiantes de medicina no creen en el juramento hipocrático, no respetan al paciente como un ser humano sino como un elemento, este entorno "termina también pasándole la factura al propio médico, ocasionándole también una desmotivación profesional, pudiendo quedar como robotizado, sometido a protocolos precisos que le protejan en caso de denuncias, y percibiendo muchas veces al enfermo como un posible enemigo" (Dr. Mario Fiore Columna Vitae, sf).

Es trascendental recalcar en este punto, que la salud física y emocional del médico es importante para un adecuado funcionamiento del servicio público de la salud, y que un ambiente laboral adecuado permite que el profesional de la salud sea más real y cercano a las necesidades del paciente, ya que si sucede lo contrario, "El paciente igualmente sobrellevará esta realidad puesto que si le falta el afecto del profesional sanitario que le atiende, su estancia en un hospital puede quedar como una amarga experiencia" (Dr. Mario Fiore Columna Vitae, s.f), sin importar en este caso la utilización de los instrumentos tecnológicos utilizados para su recuperación.

Por lo que los avances tecnológicos y científicos en el campo de la medicina, deberían traer “una medicina intensiva más humana y más justa” (Dr. Mario Fiore Columna Vitae, s.f), aquella donde se hace necesaria la humildad del profesional de la salud ante el paciente y la necesidad del conocimiento para beneficiar al enfermo, lo cual a su vez terminara beneficiando el espíritu del médico y del paciente. (Dr. Mario Fiore Columna Vitae, s.f).

\section{Conclusiones.}

Como resultado de la investigación es posible concluir que la responsabilidad médica en Colombia ha sido un tema al que se le ha restado atención, generando consecuencias negativas para la población colombiana, como vulneración de los derechos a la salud o a la vida digna, consecuencias que terminan perjudicando la salud, vida e integridad de los cientos de pacientes que acuden para ser tratados en urgencias, en controles médicos, terapias, citas prioritarias, etc. 
Colombia es un país que le falta mucho camino por recorrer en el área de responsabilidad médica, ya que su legislación carece de un desarrollo estable y continuo, haciendo que la salud no tenga un cambio de fondo que le permita mejorar efectivamente, y a pesar de poseer avances tecnológicos que podrían mejorar la calidad de vida tanto del paciente como del médico, en la práctica moderna de la medicina, la tecnología olvida la calidad de vida de los pacientes e impide un acceso igualitario, ocasionando resultados negativos como deshumanización, o estrés laboral, utilizándose no como herramienta útil sino como forma de vida en la que el paciente deja de ser un ser humano para convertirse en un "numero de cama o historia clínica".

Es importante aclarar que una de las razones principales del problema de la responsabilidad médica es la mala administración del estado, ya que tiene un enfoque erróneo que impide una prestación eficiente del servicio público, de ahí, la constante inconformidad por parte de los pacientes de todo el sistema de salud colombiano, una de las consecuencias más relevantes en una administración defectuosa por parte del estado, es la falla del servicio, que se entiende como deficiencias frente a recursos, políticas o calidad, así como un sistema en que el paciente es relegado a un tercer plano, en el que el medico se desvincula de su ética profesional, olvidando que la ética médica es su guía principal e inspiración de su conducta para evitar que con su comportamiento se vulneren los derechos de los pacientes.

Por todo esto, y por la falta de conocimiento e interés sobre la importancia de la responsabilidad médica y del manejo de la administración en la salud, es que en Colombia se genera vulneración de derechos fundamentales tanto del profesional de la salud como del paciente, y es precisamente la falta de conocimiento producto de la indiferencia lo que resulta ser el gran problema de la población, que termina contribuyendo con sus acciones a que el problema continúe sin que logre un cambio efectivo para beneficio social.

\section{Referencias:}

- Academia Nacional de Medicina en Colombia. (Sf). Impacto de las nuevas tecnologías. Documento extraído el día 20 de agosto de 2016 de 
http://anmdecolombia.net/index.php/50-home/noticias1/sesiones/273-impacto-delas-nuevas-tecnologias-sobre-los-metodos-tradicionales-de-diagnostico-ytratamiento.

- Alcaldía de Bogotá, (Sf). Ley No. 678 de 2011 Artículo 2 ${ }^{\circ}$. Por medio de la cual se reglamenta la determinación de responsabilidad patrimonial de los agentes del Estado a través del ejercicio de la acción de repetición o de llamamiento en garantía con fines de repetición. Documento extraído el día 25 de julio de 2016, de http://www.alcaldiabogota.gov.co/sisjur/normas/Norma1.jsp?i=4164

- Asociación Médica Mundial. (Sf). Manual de Ética Médica, 3º Edición, 2015. extraído el día 26 de julio de 2016, http://www.wma.net/es/30publications/30ethicsmanual/pdf/ethics_manual_es.pdf

- Asociación Médica Mundial. (Sf). Declaración de Ginebra. extraído el día 22 de junio de 2016, http://www.wma.net/es/30publications/10policies/g1/index.html

- Constitución Política de Colombia, 1991. Artículos 48 - 49 - 90

- Código de Procedimiento Administrativo y de lo Contencioso Administrativo. Ley 1437 de 2011. Artículo $3^{\circ}$, Numeral $7^{\circ}$.

- Congreso de Colombia, (Sf). Ley Estatutaria No. 1751 de 2015 Artículo 1. Por medio de la cual se regula el Derecho Fundamental a la Salud y se dictan otras disposiciones. Documento extraído el día 26 de julio de 2016, de https://www.minsalud.gov.co/Normatividad_Nuevo/Ley\%201751\%20de\%202015. pdf

- Corte Constitucional (1992) Ciro Angarita Barón. Sentencia T-406/92. Bogotá, Colombia. Extraído de http://www.corteconstitucional.gov.co/relatoria/1992/t-40692.htm

- Consejo de Estado. (2006). Documento extraído el día 25 de julio de 2016, de la base de datos de http://www.consejodeestado.gov.co/relatoriacde.php-.

- Consejo de Estado (2007). Enrique Gil Botero. Acción de reparación Directa 73001-23-31-000-1998-01327-01(17918). Bogotá, Colombia. Extraído de http://www.notinet.com.co/pedidos/17918.pdf 
- Corte Constitucional. (2015) Luis Guillermo Guerrero Pérez. Sentencia T-121/15. Bogotá, Colombia. Extraído de http://www.corteconstitucional.gov.co/RELATORIA/2015/T-121-15.htm

- Centro de bioética "Juan Pablo II" (Sf). Documento extraído el día 22 de agosto de 2016. http://www.cbioetica.org/ Documento extraído el día 22 de agosto de 2016 de

- DMedicina Salud y Bienestar. (Sf). Síndrome de Burnout. Documento extraído el día 21 de agosto de 2016, de http://www.dmedicina.com/enfermedades/psiquiatricas/sindrome-de-burnout.html

- encolombia. (Sf). Todo Sobre la Historia Clínica. Documento extraído el día 29 de junio de 2016, de https://encolombia.com/medicina/revistas-medicas/heraldomedico/vol-231/heraldo231-historia/

- Federación Médica Colombiana. (Sf). Nuestra Visión Sobre La Deshumanización De La Medicina. Documento extraído el día 20 de agosto de 2016 de http://www.federacionmedicacolombiana.org/?q=node/1004.

- Fernández M. (2008). La Responsabilidad Médica Problemas Actuales, Biblioteca de Tesis Doctorales 4. Bogotá, Colombia: Editorial Ibáñez.

- Geosalud. (Sf). Responsabilidad Medica. Documento extraído el día 22 de junio de 2016, de http://www.geosalud.com/malpraxis/respmedica.htm

- Geosalud. (Sf). La Historia Clínica: Aspectos Éticos y Legales. Documento extraído el día 28 de junio de 2016, de http://www.geosalud.com/malpraxis/historiaclinica.htm

- Gil, E. (2013). Responsabilidad Extracontractual del Estado. Sexta Edición. Bogotá, Colombia: Editorial Temis.

- Hospital Universitario San Ignacio (Sf). Definición Historia Clínica. Documento extraído el día 28 de junio de 2016, de http://www.husi.org.co/visitantes-ypacientes/historia-clinica

- Ilustrados. (Sf). El consentimiento informado en la práctica médica actual. Documento extraído el día 29 de junio de 2016, de http://www.ilustrados.com/tema/10714/consentimiento-informado-practica-medicaactual.html 
- Instituto Colombiano de Bienestar Familiar. (Sf). Manual de buen uso de la Acción de Repetición y del Llamamiento en Garantía con Fines de Repetición. Documento extraído el día 20 de julio de 2016, de http://www.icbf.gov.co/portal/page/portal/IntranetICBF/organigrama/oficinas/aseso ra_juridica/Representaci\%C3\%B3n\%20Judicial/Conciliaciones/Tab/Manual\%20bu en\%20uso\%20de\%20acci\%C3\%B3n\%20de\%20repetici\%C3\%B3n\%20y\%20llama miento\%20en\%20garant\%C3\%ADa\%20con\%20fines\%20de\%20repetici\%C3\%B3n . .pdf

- López B. (2013). Introducción al Estudio del Derecho, Importancia de las Leyes y Normas en la Sociedad, Universidad Virtual de América. Documento extraído el día 22 de $\quad$ junio $\quad$ de 2016 de https://introduccionalestudiodelderechounivia.wordpress.com/2013/11/30/importan cia-de-las-leyes-y-normas-en-la-sociedad.

- Medigraphic, Literatura Biomédica. (Sf). Síndrome de burnout en la práctica médica. extraído el día 21 de agosto de 2016 de http://www.medigraphic.com/pdfs/medintmex/mim-2012/mim126j.pdf

- Ministerio de Educación. (Sf). Ley 23 de 1981. Por la cual dictan Normas en Materia de Ética Médica. Documento extraído el día 30 de junio de 2016, de http://www.mineducacion.gov.co/1621/articles-103905_archivo_pdf.pdf

- Ministerio de Salud y protección Social. (Sf). De la Naturaleza Jurídica del derecho a la salud en Colombia. Documento extraído el día 24 de julio de 2016, de https://www.minsalud.gov.co/sites/rid/Lists/BibliotecaDigital/RIDE/IA/SSA/natural eza-juridica-derecho-salud-colombia.pdf

- Ministerio de Salud y Protección Social, (Sf). Ley Estatutaria No. 1751 de 2015 Artículo $5^{\circ}$. Por medio de la cual se regula el Derecho Fundamental a la Salud y se dictan otras disposiciones. Documento extraído el día 26 de julio de 2016, de https://www.minsalud.gov.co/Normatividad_Nuevo/Ley\%201751\%20de\%202015. pdf

- Ministerio de Salud y Protección Social (Sf). Garantizar la Funcionalidad de los Procedimientos de Consentimiento Informado. Paquetes Instruccionales. Documento extraído el día 28 de junio de 2016, de 
https://www.minsalud.gov.co/sites/rid/Lists/BibliotecaDigital/RIDE/DE/CA/Garanti $\underline{\text { zar-funcionalidad-procedimientos.pdf }}$

- Ministerio de Salud y Protección Social. (Sf). Resolución Numero 1995 de 1999. Por la cual se establecen normas para el manejo de la Historia Clínica. Documento extraído el día 30 de junio de 2016, de https://www.minsalud.gov.co/Normatividad_Nuevo/RESOLUCI\%C3\%93N\%2019 95\%20DE\%201999.pdf

- Ministerio de Salud y Protección Social. (Sf). Estudio sobre el modo de gestionar la salud en Colombia. Documento extraído el día 22 de agosto de 2016, de https://www.minsalud.gov.co/Documentos\%20y\%20Publicaciones/Estudio\%20sobr e\%20el\%20modo\%20de\%20gestionar\%201a\%20salud\%20en\%20Colombia.pdf

- MundoGestalt. Artículos de la Universidad Gestalt. (Sf) Importancia de la Historia Clínica. Documento extraído el día 30 de junio de 2016, de http://mundogestalt.com/importancia-de-la-historia-clinica

- Nisimblat Abogados. (Sf). Responsabilidad Medica. Documento extraído el día 23 $\begin{array}{llll}\text { de } & \text { junio } & \text { de }\end{array}$ http://nisimblat.net/responsabilidadmedica/faqsrespmedica.htm

- Osorio, A. (2002). Breve aproximación a algunos aspectos de la responsabilidad penal médica, Trabajo de Grado para optar al título de Abogado, Pontificia Universidad Javeriana. Documento extraído el día 22 de junio de 2016, de http://www.javeriana.edu.co/biblos/tesis/derecho/dere4/Tesis-14.pdf

- Periódico El Espectador. (Sf). Los tres males del sistema de salud. Documento extraído el día 22 de agosto de 2016, de http://www.elespectador.com/noticias/salud/los-tres-males-del-sistema-de-salud$\underline{\operatorname{articulo}-361405}$

- Periódico La Nación. (Sf). Deshumanización en salud. Documento extraído el día 23 de agosto de 2016, de http://www.lanacion.com.co/index.php/opinion/item/220236-deshumanizacion-ensalud

- Psico Ayuda. (Sf). Despersonalización en "burnout”. Documento extraído el día 21 de agosto de 2016, de http://psicoayuda.enredado.org/?p=25 
- Psicología y Mente. (Sf). Burnout (Síndrome del quemado): cómo detectarlo y tomar medidas. extraído el día 21 de agosto de 2016, de https://psicologiaymente.net/empresa/burnout-sindrome-del-quemado

- Red Jurista, (Sf). Sentencia Corte Constitucional, 1992. Documento extraído el día 27 de julio de 2016, de https://www.redjurista.com/documents/66001-23-31-0001998-00181-01(24985).aspx Corte Constitucional, 1992

- Rodríguez. L. (2013). Derecho Administrativo General y Colombiano. 18 edición Bogotá, Colombia: Editorial Temis S.A.

- Scielo, Scientific Electronic Library Online. (Sf). Consentimiento informado: algunas consideraciones actuales. Documento extraído el día 29 de junio de 2016, de http://www.scielo.cl/scielo.php?script=sci_arttext\&pid=S1726$\underline{569 \times 2012000100011}$

- Scielo, Scientific Electronic Library Online. (Sf). Síndrome de burnout en médicos y personal paramédico. Documento extraído el día 22 de agosto de 2016, de http://www.scielo.cl/scielo.php?script=sci_arttext\&pid=S1726569X2010000200004.

- Scielo, Scientific Electronic Library Online. (Sf). Calidad de Atención y Principios Éticos. Documento extraído el día 23 de agosto de 2016, de http://www.scielo.cl/scielo.php?script=sci_arttext\&pid=S1726569X2010000200004.

- Senado de la República, Sala de Prensa. (Sf). La Paradoja de la Salud en Colombia. Documento extraído el día 23 de agosto de 2016, de http://senado.gov.co/sala-deprensa/opinion-de-senadores/item/14494-la-paradoja-de-la-salud-encolombia?tmpl=component \&print $=1$.

- Sura Blog (Sf). Salud: consentimiento informado Un derecho para ejercer. Documento extraído el día 29 de junio de 2016 de http://www.sura.com/blogs/calidad-de-vida/consentimiento-informado.aspx

- UNAD - Universidad Nacional Abierta y a Distancia. (Sf). Lección 3: Falla en el Servicio por parte de la Administración. Documento extraído el día 20 de junio de 2016, 
http://datateca.unad.edu.co/contenidos/109133/eXe_109133/Modulo/MODULO_E $\underline{\text { XE/leccin_3 falla_en_el_servicio_por_parte_de_la_administracin.html }}$

- Universia Colombia. (Sf). Administración en salud, el lunar en el Servicio en Colombia. Documento extraído el día 23 de agosto de 2016, de http://noticias.universia.net.co/actualidad/noticia/2014/04/10/1094735/administracio $\underline{\text { n-salud-lunar-servicio-colombia.html }}$

- Universidad del Rosario - Universidad CES. Facultad de Medicina Especialización en Epidemiología. (Sf). Síndrome de burnout y factores asociados en personal de salud en tres instituciones de Bogotá en diciembre de 2010. Documento extraído el día 23 de agosto de 2016, de http://repository.urosario.edu.co/bitstream/handle/10336/2389/529311431.pdf? sequence $=1-$ 